\title{
INVESTIGACIONES
}

\section{Hacer de lo familiar extraño: análisis de un ejercicio docente respecto a las creencias y concepciones que profesoras y profesores que enseñan ciencias tienen sobre su labor pedagógica}

Make the familiar strange: analysis of a teaching exercise regarding the beliefs and conceptions that teachers who teach science classes have about their pedagogical work

\section{Marta Silva Fernández ${ }^{a}$, Jennifer Diana Brito Pacheco ${ }^{b *}$, Paulina Danisa Larrosa López, Paulina Constanza Sanzana Müller ${ }^{d}$, Tamara Paola Busquets Püschel ${ }^{e}$}

\author{
${ }^{a}$ Ph.D en Educación, Universidad Austral de Chile. \\ marta.silva@uach.cl \\ ${ }^{b}$ Magíster en Educación, Universidad Austral de Chile. \\ jenniferdianabrito@gmail.com \\ ${ }^{c}$ Dra. en Ciencias Humanas mención discurso y cultura, Universidad Austral de Chile. \\ paulina.larrosa@uach.cl \\ ${ }^{d}$ Licenciada en Lenguaje y Comunicación, Colegio Gracia y Paz. \\ paulisanzana@gmail.com \\ ${ }^{e}$ Doctorado en Ciencias Naturales, Universidad Austral de Chile. \\ tamara.busquets@uach.cl
}

\section{RESUMEN}

La literatura respecto a las creencias y concepciones que las y los profesores tienen sobre sus prácticas pedagógicas es vasta, tanto a nivel nacional como internacional, no obstante, en el contexto chileno, aún quedan algunos aspectos por conocer, particularmente en el ámbito de la enseñanza y aprendizaje de las ciencias, donde la literatura es escasa. El presente estudio cualitativo analizó 28 testimonios de profesores que imparten clases de ciencias en la Región de los Ríos, para conocer la percepción que tienen de sus propias prácticas pedagógicas por medio de un ejercicio denominado extrañamiento, en el cual debieron explicar a un ente extraterrestre que desconocía la labor de un profesor, en qué consistía su profesión y qué significaba para ellas y ellos. El análisis de contenido reveló que sus concepciones varían entre un polo tradicional de la enseñanza y un polo innovador basados en un enfoque constructivista de la educación. Los resultados demuestran una concepción más constructivista de la enseñanza que conductista, mostrando a su vez, que existe un trabajo colaborativo dentro de la comunidad educativa.

Palabras clave: creencias y concepciones docentes, enseñanza de las ciencias, extrañamiento, enfoques de enseñanza.

\section{ABSTRACT}

The literature regarding the beliefs and conceptions that teachers have about their pedagogical practices is vast, both nationally and internationally, however, in the Chilean context, there are still some aspects to be known, particularly in the field of teaching and science learning where literature is almost non-existent. The present qualitative study analyzed 28 testimonies of teachers who teach science classes in the Los Ríos Region, to know the perception that have of their own pedagogical practices through an exercise called estrangement, in

* Becaria de la Agencia Nacional de Investigación y Desarrollo de Chile- ANID, Doctorado Nacional 2019, folio 21192056. 
Estudios Pedagógicos XLVII N 3: 27-43, 2021

HACER DE LO FAMILIAR EXTRAÑO: ANÁLISIS DE UN EJERCICIO DOCENTE RESPECTO A LAS CREENCIAS Y CONCEPCIONES QUE PROFESORAS Y PROFESORES QUE ENSEÑAN CIENCIAS TIENEN SOBRE SU LABOR...

which had to explain to an extraterrestrial entity that did not know the work of a teacher, what their teaching profession consisted of and what it meant for them. The content analysis revealed that their conceptions vary between a traditional pole of teaching and an innovative pole based on a constructivist approach to education. The results demonstrate a more constructivist than behavioral conception of teaching, showing in turn that there is collaborative work within the educational community.

Key words: teaching beliefs and conceptions, science teaching, estrangement, teaching, approaches.

\section{MARCO TEÓRICO: CONTEXTUALIZACIÓN}

En Chile los avances en las tecnologías y en la ciencia en general, constituyen un progreso en las condiciones y originan nuevas formas de vida en sociedad. Sin embargo, estas transformaciones también han generado diferentes problemáticas, como la escasez de recursos naturales, aumento del cambio climático, destrucción de la flora y fauna nacional.

Sobre ello, se ha argumentado que la educación y los educadores deben convertirse en líderes, actores reflexivos y autónomos, pues está en sus manos el futuro de las nuevas generaciones. Más aún, se ha escuchado o leído en los medios de comunicación y redes sociales, sobre la estrecha relación entre calidad, equidad y resultados de aprendizaje. Así, también las políticas públicas en el área educativa, han hecho eco a las demandas sociales y las necesidades de mejora en la cuestión docente. Al respecto, la política originada el año 2016 que crea el sistema de desarrollo profesional docente, reconoce la importancia de estos profesionales para el país y diseña un marco legislativo que busca su revalorización social (Ruffinelli, 2016).

En consideración a lo anterior, la profesión docente se reconstruye permanentemente, sustentada en los marcos sociales, culturales e históricos presentes en un determinado tiempo y lugar. A su vez, estos elementos se vinculan a las definiciones conceptuales, epistemológicas y emotivas de los propios profesores. Acorde a estos planteamientos, Ávalos y Sotomayor (2012) mencionan la relevancia de indagar en los mundos de significados y sentidos de estos actores. Es decir, descubrir sus motivaciones, creencias sobre su desempeño profesional, los componentes a los que atribuyen mejoras en su labor, así como aquellos incidentes críticos, que viven a lo largo de sus recorridos profesionales.

En el caso de los profesores de ciencias, Busquets et al. (2016) señalan que se les solicita un rol preponderante en el desarrollo del pensamiento crítico, la innovación y en la creación de nuevas soluciones en los contextos escolares. Subrayando, que su formación profesional debería considerar sus conocimientos disciplinares, sus contextos socio-históricos y una gran diversidad de saberes provenientes de las ciencias de la educación. Junto a sus propias experiencias y reflexiones aprehendidas en sus vidas. Para ello, sus saberes disciplinares y pedagógicos; sus metodologías y estrategias de enseñanza-aprendizaje; características personales y profesionales, deben propender al desarrollo de las denominadas habilidades blandas o competencias para el siglo XXI (Reimers y Chung, 2016). Así mismo, realizando su quehacer profesional apoyados por los enfoques educativos constructivistas, en donde la indagación se convierta en una herramienta y bandera de primera línea (Busquets et al., 2016).

Aunque en las realidades, muchos de estos desafíos son compartidos o se diluyen en las propias creencias y concepciones de los profesores, sobre lo que implica el proceso de enseñanza-aprendizaje y su labor en las escuelas en que trabajan. En este sentido, se 
considera que develar dichas creencias y concepciones ponen de manifiesto su pensamiento pedagógico en el contexto de las clases de ciencias. Tal, como se presenta en los siguientes apartados.

\section{COGNICIONES DOCENTES SOBRE LA ENSEÑANZA-APRENDIZAJE Y SU LABOR PROFESIONAL}

Las investigaciones sobre profesores en las últimas décadas han valorado desde diferentes perspectivas su participación y rol en las comunidades escolares, considerando sus pensamientos, capacidades y acciones en el contexto escolar (Shulman, 1989).

Sobre lo anterior, Barrón (2015) menciona que los primeros estudios sobre docentes en el siglo XX buscaban definiciones de su labor profesional desde una mirada instrumentalista y racionalista. En esta línea, los educadores son considerados técnicos especialistas, cuya meta final es conseguir la eficacia-efectividad de sus clases. En otras palabras, el énfasis se encuentra en los resultados de aprendizajes de sus estudiantes, por medio de la relación causal proceso-producto. Dicha vinculación, se explica en parte, por los postulados del enfoque del condicionamiento y las lógicas de las aulas como laboratorios de enseñanza (Shulman, 1989). En esta mirada teórica, se destaca la supremacía de los educadores, quienes se convierten en el instrumento utilizado para hacer entender los conceptos, procesos y contenidos en las mentes vacías de sus estudiantes. Así, surge la relevancia del rendimiento académico, como meta final a alcanzar. La pedagogía en este sentido, pasa a ser un fenómeno visible y cuantificable, que entrega validez y confiabilidad a las acciones de los actores educativos, en especial de los enseñantes (Schunk, 2012).

Como contraparte a estos planteamientos, surge en la década de los 70' el enfoque del pensamiento del profesor. Este, en un primer momento fue vinculado con las visiones cognitivistas que indagaban en las variables de rendimiento y eficacia escolar (Shulman, 1989). Aunque con el correr del tiempo fue reconfigurándose hacia la comprensión de cómo los educadores piensan, conocen y realizan sus valoraciones (Echeverría et al., 2006) y rutinas (Lowyck, 1988). Sumado, al estudio de las construcciones subjetivas que ocurren en su mente, desarrolladas por medio de procesos psicológicos que organizan los comportamientos que realizan en la enseñanza. Sobre ello Lowysck (1988), señala que los enseñantes no son solo sujetos racionales y que muchas de las acciones que realizan las desarrollan de forma intuitiva.

Coincidiendo con estas ideas Imbernón (2007), manifiesta la relevancia de investigar a los profesores "desde el punto de vista de sus sentimientos, decisiones, creencias y pensamientos, y de su influencia en la acción educativa" (p. 127). Para el autor, esto surge al vincular las intenciones de los educadores con sus quehaceres y vida cotidiana. Estas permiten cimentar su formación, desarrollo profesional y la innovación en su campo de acción, es decir, la escuela. Dicho de otra manera, las cogniciones de los profesores nutren las teorías y creencias que tengan sobre la enseñanza en general y en su propio desempeño. Junto a la manera en que ordenan y anticipan su enseñanza, en la planificación y en la toma de decisiones que desarrollan, atendiendo las interacciones en un contexto y momento determinado (Echeverría et al., 2006).

Sin embargo, no es hasta los desarrollos teóricos de Shön (1992), que los docentes son considerados por los investigadores como sujetos reflexivos, autónomos, que interpretan la 
realidad desde sus contextos psicológicos. En concreto, estos se encuentran conformados por sus teorías implícitas, creencias y valores sobre los significados de la enseñanza y el aprendizaje, por lo que no solo alude a los conocimientos y saberes que culturalmente se les han atribuido, sino que busca sus meta-cogniciones (Barrón, 2015). Estas últimas, entendidas como los análisis que desarrollan sobre la naturaleza y componentes de la enseñanza. Además, de la influencia de los entornos escolares en sus ideas, formas de ver el mundo y su trabajo. Sumado al dominio de dichos elementos en sus decisiones, prácticas pedagógicas e interacciones, que desarrollan con los otros actores de las comunidades educativas.

En este sentido, Marcelo y Vaillant (2013) plantean que las experiencias escolares y las interpretaciones que los profesores realizan sobre ello, tienen impacto en su presente y futuro. En particular, sobre las maneras de enseñar y evaluar los aprendizajes. Dado que sus propios profesores y las interacciones pretéritas con los entornos escolares, se instalan en sus trayectorias contribuyendo a su identidad profesional. En otras palabras, se comienzan a forjar y desmontar sus significaciones sobre el ser profesor, desde los momentos previos a la formación oficial que reciben en los programas de pedagogía.

Así mismo, se constata que la profesión docente se encuentra entretejida por la praxis, con saberes experienciales entre sus miembros (Tardif, 2010). Estos, organizan un cúmulo de representaciones compartidas, que les permiten entender y guiar en su labor a aquellos educadores que se incorporen en las escuelas o que inician su ejercicio profesional. Por lo tanto, creando una cultura docente situacional, que es retroalimentada por las preconcepciones, suposiciones y definiciones que cada uno de ellos tenga sobre la forma correcta de actuar y desarrollar la docencia en las instituciones escolares. Sobre ellas, cabe señalar que son difundidas en los espacios de diálogo y reunión profesional. Específicamente en aquellas de tipo formal (los clásicos consejos docentes o las actuales comunidades de aprendizaje) e informales (por ejemplo, conversaciones en los denominados recreos, espacios de tiempo entre un segmento y otro de clases).

\section{CREENCIAS Y CONCEPCIONES DOCENTES}

Como ya hemos señalado, dentro de los contextos psicológicos de los enseñantes encontramos sus creencias. Estas son entendidas como un cúmulo de ideas verdaderas que poseen los profesores sobre el fenómeno educativo, que les permite comprender, entender, interpretar la enseñanza-aprendizaje y sus dinámicas en el entorno en que ocurren (Hidalgo y Murillo, 2017; Inguanzo, 2010; Pajares, 1992). Así mismo, estas preconcepciones se caracterizan por ser subjetivas y poseer dimensiones emocionales-afectivas, cognitivas y conductuales sustentadas en sus marcos epistemológicos. Éstos, son construidos en su recorrido profesional (Inguanzo, 2010) e "influyen en la forma en que se utiliza, almacena y recupera el conocimiento" (Marcelo y Vaillant, 2013, p. 68), impactando en la propia imagen que tienen los maestros de sí mismos y de su trabajo cotidiano (Díaz et al., 2010).

Coincidiendo con estos planteamientos, Pajares (1992) agrega que para aclarar el panorama conceptual en los estudios sobre creencias de las y los enseñantes, se debe considerar que estas corresponden a valorizaciones personales. Por consiguiente, a diferencia del conocimiento, no necesitan evidenciar si son verídicas o no, pues para quién las produce son premisas incuestionables. Sin embargo, Garritz (2014) manifiesta que ha 
resultado una tarea compleja llegar a consensos sobre ello, ya que tanto el conocimiento como las creencias se encuentran en un diálogo permanente. Sumado a que estas últimas, actúan como una hoja de ruta para explicar los nuevos acontecimientos, que le ocurran al docente (Hidalgo y Murillo, 2017). Cabe destacar, que todo esto ocurre bajo una carga emocional importante, pero sin evidencias tangibles e irrefutables. Es decir, solo se sustentan en lo que cada educador piensa sobre algo o alguien, por lo que se encuentran arraigadas en su sentido común, y, son difíciles de cambiar a menos que se hagan visibles. Además, porque se establecen en límites difusos, entre los saberes disciplinarios y experienciales de dichos profesionales (Díaz et al., 2010).

Por su parte Echeverría et al. (2006), sostienen que los enseñantes poseen creencias epistemológicas cotidianas, que abordan las preguntas y respuestas sobre qué significa el conocimiento y sus diversas maneras de enseñarlo. Así, existirían creencias que buscan validar el conocimiento entre un rango absoluto y relativo, originadas en las dimensiones externas e internas del sujeto. En relación a las primeras, se debe señalar que surgen desde los entornos de los educadores y se fundamentan en evidencias científicas. Mientras que, en las segundas las y los profesores son creadores de nuevos conocimientos, que nacen de sus justificaciones personales (Shunk, 2012).

En cuanto a las concepciones, Ramos y Casas (2018) establecen que los componentes del conocimiento de los enseñantes refinan "la toma de decisiones, son producto del entendimiento e influyen en los procesos de razonamiento" (p. 4), constituyéndose como una estructura ordenada de creencias. Estas provienen de las vivencias de los sujetos y son puestas en escena, por medio de las interacciones que realizan en los diferentes lugares en que se desenvuelven. En concreto, se presentan como una manera que poseen los educadores de comprender la enseñanza y aprendizaje. Es decir, como interpretan su campo de actuación o práctica profesional de acuerdo a sus experiencias de vida y a las comunidades educativas en que se desempeñan (Hidalgo y Murillo, 2017).

Con respecto, a las creencias y concepciones de los educadores en el contexto chileno, es posible señalar que han sido ampliamente investigadas. Contribuyendo, de acuerdo a Ruiz et al. (2014) a la creación de un marco de estudios que ayuda a "...orientar propuestas de intervención que involucren a estos docentes en la comprensión y regulación de las relaciones entre su pensamiento y su desempeño en el aula" (p. 55). En esta lógica, algunos hallazgos interesantes en dichas investigaciones, revelan la existencia de divergencias entre las concepciones y creencias de los docentes sobre la enseñanza, el aprendizaje y la evaluación educativa (Arancibia-Herrera et al., 2019) de acuerdo al nivel educativo (Guerra y Sebastián, 2015) y a las disciplinas en que realizan su trabajo (Vizcaino et al., 2015). Así, por ejemplo, Guerra y Sebastián (2015) realizaron un estudio sobre las creencias epistemológicas de profesores chilenos, que postulan a un programa de incentivo y reconocimiento profesional. Entre los resultados de esta investigación, destaca que "conciben el conocimiento como piezas aisladas unas de otras y que permanecen estables en el tiempo" (p. 120), por lo que predomina una visión conductista e instrumentalista de la enseñanza-aprendizaje. Junto con las demandas externas de la sociedad chilena de transformación y generación de conocimiento situado.

De igual manera, de acuerdo al nivel de enseñanza en que los educadores realizan sus quehaceres, algunos investigadores (Arancibia-Herrera et al., 2019; Ravanal et al., 2018; Vizcaino et al., 2015), coinciden en que aquellos de nivel secundario poseen creencias y concepciones sobre su labor, con un mayor realce disciplinar. Esto se puede explicar, 
por la formación inicial especializada que recibieron en un área de las ciencias sociales, humanas, naturales o matemáticas. También, se evidencian diferencias de acuerdo a la participación de los docentes en programas formales de desarrollo profesional (cursos, programas de magíster y doctorado) y a la etapa de la docencia en que se encuentren (Guerra y Sebastián, 2015).

En esta lógica, en un estudio en que participan profesores de educación secundaria con especialidad en matemáticas, se concluye que las concepciones y creencias de dichos profesionales, conciben la enseñanza bajo los postulados teóricos de la disciplina. Es por ello, que su quehacer principal estaría en preparar las actividades y realizar ejercicios para desarrollar sus clases. Aunque, el aprendizaje es presentado desde aspectos cognitivos que deben ser potenciados, tanto por el propio estudiante como por otra persona. Esto, a través de diferentes acciones de apoyo, por lo que cualquier estudiante que cuente con el interés personal y la ayuda necesaria podría desempeñarse de buena forma en esta asignatura. Es decir, existirían contradicciones en las concepciones y creencias de los educadores entre los enfoques educativos conductistas y contructivistas (Donoso et al., 2016).

\section{CREENCIAS Y CONCEPCIONES DE DOCENTES DE CIENCIAS NATURALES SOBRE EL PROCESO DE ENSEÑANZA-APRENDIZAJE}

Como se ha presentado en los apartados anteriores, el estudio de las creencias y concepciones que poseen los educadores sobre la ciencia y su enseñanza, es un tema relevante para comprender las decisiones y acciones que desarrollan en las aulas. En esta línea, se presentan algunas investigaciones en las que participan enseñantes, en diferentes momentos de sus trayectorias profesionales.

En relación a la formación inicial, García y Acevedo (2016) realizan un estudio cuantitativo en una universidad española que indaga en las concepciones sobre la naturaleza y didáctica de las ciencias, para estudiantes de un programa de nivel primario. Entre sus resultados, sostienen que las concepciones sobre la ciencia para los futuros profesores, se encuentran en constante trance. Esto se explicaría, porque los participantes creen que los conocimientos irrefutables de antaño, ya no se pueden dar por sentado, producto de los cambios socio-culturales y económicos que ocurren a nivel global y local. Además, argumentan la existencia de supuestos epistemológicos y ontológicos tras las decisiones teóricas de los científicos. Por lo tanto, cuestionan el axioma de posición neutral, objetiva, explicativa de las ciencias modernas y empíricas.

Profundizando en este último punto, Orellana et al. (2018) en una investigación con estudiantes de educación inicial en el contexto chileno, argumentan que poseen concepciones de enseñanza-aprendizaje "que se pueden asociar tanto a un Racionalismo Radical y una Noción Tradicional-Dogmática de la enseñanza y el aprendizaje de las Ciencias, como a un Racionalismo Moderado y una noción constructivista del mismo" (p. 1038). En otras palabras, cohabitan en sus cogniciones, perspectivas que pueden resultar dispares en sus fundamentos teóricos-epistemológicos y finalmente en las prácticas que realicen en las escuelas.

En docentes en ejercicio, Schunk (2012) refiere que sus concepciones y creencias ocurren desde dos vertientes. La primera, con posiciones epistemológicas tradicionales sustentadas en el racionalismo y la experimentación, en donde ellos son encargados de 
trasmitir los conocimientos de las disciplinas ante estudiantes pasivos que reproducen los saberes adquiridos, bajo el enfoque conductista. De manera puntual, su disciplina o el contenido que enseñan, es el principal motor de sus prácticas pedagógicas. Más aún, dichas cogniciones actúan como filtro en la planificación de su enseñanza, en sus interacciones con los otros actores educativos y en síntesis en su propio desarrollo profesional. Este último, materializado, por ejemplo, en las elecciones que realizan de cursos de perfeccionamiento o postgrados (Garritz, 2014).

En la segunda vertiente, la ciencia es visualizada como construcción socio-cultural de negociaciones entre sus miembros. Para ello, el diálogo juega un rol fundamental, precedido por la valorización del sujeto que aprende, sus cogniciones, posibilidades de generación y construcción de sus saberes (Shunk, 2012). De modo que, se evidencian posiciones constructivistas sobre lo que piensan de la naturaleza del conocimiento y las formas de realizar su labor. Estas, se plasman en la planificación, gestión de la enseñanza, estrategias, recursos didácticos y evaluaciones educativas (Ruiz et al., 2014).

Por su parte, Fernández et al. (2009) efectúan un estudio con profesores de enseñanza primaria de México, para relacionar sus concepciones con las prácticas pedagógicas presentes en sus clases de ciencias. Los autores, identifican tres concepciones fundamentales: tradicional, transición y constructivistas. La primera de ellas, concibe la enseñanza como instrucción, en donde el profesor como personaje central entrega informaciones y habilidades a sus estudiantes, por lo que el aprendizaje es concebido como una repetición con tareas rutinarias. En aquellas de transición, la premisa es "se aprende practicando", por lo que la enseñanza procura desarrollar diferentes aspectos en los estudiantes de manera activa. En tercer lugar, las concepciones constructivistas se encuentran centradas en el aprendizaje. De modo que, los estudiantes al interactuar con sus ambientes, desarrollan conocimientos situados y significativos. Esta concepción, reconoce a las y los estudiantes como: personas integrales, con un mundo emocional, con capacidades para reflexionar sobre su propio aprendizaje y elaborar relaciones complejas, entre los conocimientos que se les presentan y aquellos que han adquirido a lo largo de sus biografías.

Finalmente, en Chile, específicamente en la región Metropolitana y Coquimbo, Ravanal et al. (2018) indagan sobre las creencias de profesores que imparten la asignatura de biología, al preparar y evaluar el proceso de enseñanza-aprendizaje. Con respecto a sus hallazgos, los investigadores destacan diferencias consustanciales, entre los pensamientos que verbalizan los educadores y sus prácticas pedagógicas. Así, aunque muchas de sus enunciaciones responden a los planteamientos constructivistas, en su ejercicio profesional se presentan razonamientos y conceptos marcados por la disciplina científica que imparten, es decir, por la perspectiva tradicional.

\section{METODOLOGÍA}

Este estudio de carácter cualitativo (Danzin y Lincon, 2011) busca la profundidad de las creencias y concepciones de las y los docentes de la región de los Ríos sobre su labor en la enseñanza de las ciencias. Para ello, se realiza un ejercicio de extrañamiento, que es entendido como una actitud científica con la que se mira una realidad cultural (Lins Ribeiro, 2004). De igual modo, en el campo educativo el extrañamiento es utilizado, "como herramienta metodológica de carácter cognoscitivo [...], permite no ignorar aquello que es para nosotros 
irracional y/o incomprensible desde los patrones culturales propios. Es decir, no incurrir en análisis etnocéntricos" (Olmos, 2016, p. 5). Por el contrario, admite una observación consciente de la propia vida o del entorno, en donde no hay seres o culturas superiores.

En el caso de este estudio, el ejercicio de extrañamiento, se constituye como una posición reflexiva: los profesores hacen de lo "familiar algo extraño". Para ello, imaginan un encuentro con un alienígena, un ser que desconoce sus marcos cognitivos, sociales, culturales y profesionales, por lo que desde sus experiencias lo acercan a las implicancias de su labor. Luego, realizan un escrito a mano en una hoja de papel frente a la pregunta "Si se encontrara con un ser extraterrestre que no conoce absolutamente nada de la Tierra, y particularmente del trabajo de los profesores, ¿Cómo explicaría usted a este personaje su labor como docente?". Los educadores tuvieron un máximo de 45 minutos para responder.

En relación a los participantes, se debe señalar que correspondían a 28 profesoras y profesores que imparten clases de ciencias, desde el nivel preescolar hasta el secundario, con experiencia laboral de entre 2 y 25 años. En este punto, cabe indicar que estos profesionales asistieron a un curso sobre la metodología de indagación en ciencias y que una de las actividades que realizaron en esta ocasión, correspondía al ejercicio de extrañamiento, anteriormente descrito.

Una vez recolectados los testimonios, éstos fueron transcritos en un procesador de textos. Posteriormente, fueron leídos en reiteradas ocasiones en busca de grandes categorías que se repitieran, a través del análisis de contenido. Luego, se elaboraron tablas, las cuales daban cuenta de los principales temas y subtemas de cada uno de los documentos (Miles y Huberman, 1994; O'Reilly y Parker, 2014). Todas estas categorías se dividieron en subcategorías, que fueron validando las ideas y temáticas fuerza del estudio.

Finalmente, es importante mencionar, que en las textualidades presentes en el siguiente apartado de resultados y discusión, los nombres de las y los profesionales han sido cambiados, siguiendo las normas de ética de la investigación, resguardando el anonimato de los participantes. Además, se le ha asignado un número a cada uno de sus textos o testimonios $(\mathrm{T} 1,2,3 \ldots)$, junto con su identificación en el nivel de enseñanza en que realizan sus clases de ciencias (n. preescolar, primario o secundario).

\section{RESULTADOS}

\subsection{LABOR DOCENTE}

\subsubsection{Finalidad de la enseñanza: Importancia del trabajo de los docentes en el aprendizaje de las y los estudiantes}

En aula, la labor docente posee un rol de gran importancia, pues son las y los profesores, quienes posibilitan el vínculo entre la enseñanza y el aprendizaje. Es por esta razón, que en esta investigación se indagó acerca de la labor docente y sus implicancias en la educación, a través del análisis de cartas o testimonios, que los profesores le escribieron a un extraterrestre, mediante un ejercicio de extrañamiento. Junto a ello, se analizó también, la concepción que los educadores tienen respecto a la adquisición de los nuevos conocimientos en sus estudiantes. 
A partir de lo anterior, algunos profesores indicaron que la finalidad de la enseñanza es educar. En el caso concreto, de (Pedro, T2, n. secundario: "educar, es invitar a los niños y niñas a aprender de sí mismos y del entorno que los rodea, así de distintas maneras vamos adquiriendo nuevos conocimientos, y lo principal, comprendiéndonos a nosotros mismos y a los demás". Acorde a estas ideas, Maritza (T10, n. primaria) manifiesta que enseñar es: "entregar herramientas que permitan conocer el mundo que nos rodea y también a nosotros mismos, todo esto con el propósito de que cada ser humano logre encontrar su lugar en este mundo". Mientras que María piensa que:

enseñar, esto significa entregar conocimientos, desarrollar también las capacidades y habilidades que los niños traen desde sus hogares. Además, se desarrollan en ellos valores, que se van trabajando durante las mismas actividades, estos valores pueden ser, por ejemplo, respeto a los demás, solidaridad, compañerismo, etc. A lo cual se suma, la idea de que los niños y niñas pequeños aprenden por medio del juego, y en ocasiones también a través de la socialización e investigación (T27, n. preescolar).

Todo lo cual se conjuga para explicar el propósito de la enseñanza, al complementarse con la visión de las y los docentes, sobre su labor como guía y de la percepción que los educandos tienen de su propio aprendizaje y de sus profesores. Al respecto, un enseñante (Mario, T3, n. secundario) que: "el ser educadores implica enseñar diferentes cosas, a fin de que los estudiantes amplíen la mirada del mundo que los rodea"). Asimismo, la profesora Carmen, quien es educadora de párvulos (T8, n. preescolar), indicó que: "la misión que tenemos las educadoras es instruir y dar sentido al trabajo diario de los niños", lo cual implica que el docente no solo transmite conocimientos, sino también es un puente que permite que los educandos experimenten, a fin de que, en palabras de (Pedro, T2, n. secundario): "de forma individual y compuesta, se pregunten sobre lo que están viendo y sintiendo".

Ahora bien, en relación a la percepción que los estudiantes tienen de sus profesores, se destaca la visión de madre que varias de las profesoras resaltaron al explicar su rol como docentes. En palabras de la profesora Mónica (T5, n. primaria):

muchas veces uno pasa a ser la segunda madre de los niños, de hecho, algunos se equivocan y nos dicen mamá, pues pasamos muchas horas con ellos, es por esta razón que también me doy cuenta cuando ellos están tristes o alegres.

En palabras simples, según la profesora Laura (T17, n. preescolar), como docentes cumplimos la función de una segunda madre, ya que nos preocupamos del cuidado integral de nuestros alumnos, entregándoles protección y amor. Este vínculo proporciona bienestar y seguridad entre los estudiantes quienes se sienten a gusto a la hora de aprender. Así lo señala la profesora Mónica (T5, n. primaria): "en la sala de clases las y los estudiantes tienen la posibilidad de interactuar y adquirir nuevos conocimientos, utilizando variados materiales, además de enseñar valores y modales". Se suma a lo anterior, lo declarado por la profesora Elena (T7, n. primaria), quien indicó que los alumnos se sienten cómodos en el aula, ya que para ellos es "como ir a jugar porque tienen allí espacio para recorrer, para conversar y crear nuevas cosas, puedan además entender cómo funciona un poco eso llamado vida, donde ellos construyen, arman, investigan, exploran, comparten y se forman vínculos”. 


\subsubsection{Visiones disimiles acerca de la concepción de enseñanza-aprendizaje}

En relación a la concepción de enseñanza y aprendizaje, en general las y los docentes concordaron en que esta es una tarea recíproca, en donde, por un lado, las y los estudiantes se motivan a adquirir nuevos conocimientos, a través de, actividades teórico-prácticas. Mientras, que por otro, los educadores, utilizan diversas herramientas didácticas para instar a sus alumnos a estudiar el área de las Ciencias; esto se puede constatar en el relato de la profesora Claudia (T12, n. preescolar) cuando dice:

En mi planeta Tierra, entregamos conocimientos a los niños y niñas a través de la educación, [...], yo principalmente entrego educación (conocimiento), valores, hábitos, etc. a los niños y niñas pequeños, donde el juego tiene un rol fundamental, el cual se lleva a cabo también a través de la socialización o investigación. Mi rol en mi trabajo es de aula, activa y efectiva, modeladora, logrando que los niños y niñas tengan confianza y que mis aprendizajes sean cercanos a su realidad y entorno.

Asimismo, la profesora Emma (T18, n. primaria) hizo alusión a que ella es una mediadora, contribuyendo a la adquisición de nuevos conocimientos, donde los estudiantes son los actores principales al aprender haciendo, la profesora lo ejemplifica al recordar la opinión que un estudiante tiene de la labor docente,

La profesora es la persona que esta diariamente en este lugar, la que nos enseña, nos da a conocer en que trabajaremos hoy, y nosotros los alumnos damos opiniones, respetamos al otro, investigamos, indagamos sobre el tema, trabajamos en equipo, tratamos de ayudarnos, si a un compañero le falta material, si le cuesta entender lo ayudamos, realizamos experimentos para comprobar lo que estamos aprendiendo.

Lo anterior se complementa por lo expuesto por la profesora Luisa (T23, n. preescolar) quien manifestó que para ella es fundamental pedir la opinión de sus estudiantes, pues siente que, al realizar esta acción, las y los alumnos perciben que son parte de la planificación de sus clases. Por lo que, se generan las condiciones para que exista un trabajo activo y colaborativo por parte de las y los estudiantes.

A diferencia de lo descrito con anterioridad, la profesora Antonia (T14, n. secundario) relató que ella utiliza otra metodología para transmitir el conocimiento a sus estudiantes, "en general creo que muchas de las clases que hago responden a una clase tradicional, donde el estudiante escucha la restricción del profesor y responde la actividad, en general trato de ser diferente pero lo tradicional gana". Estas dos miradas, constituyen los dos polos, a través de los cuales se enseña, y esto también determina la forma de recepción del conocimiento, una será pasiva y la otra activa, a través del aprender haciendo. Sin perjuicio de esto, ambas posturas, sin importar la forma como se trasmite el conocimiento, tienen como eje central el desarrollo de capacidades, habilidades y valores entre sus estudiantes, así lo corrobora lo relatado por la profesora Fabiola (T28, n. preescolar): "como docente, desarrollo en ellos valores que se van trabajando durante las mismas actividades que les hago día a día, estos valores pueden ser por ejemplo respeto a los demás, solidaridad y compañerismo, entre otros valores. 


\subsection{CONTEXTO EDUCATIVO}

\subsubsection{Escuela: Espacio Educativo donde la Comunidad Educativa trabaja colaborativamente}

La enseñanza y el aprendizaje se llevan a cabo en el espacio educativo, el cual contempla la estructura física donde se imparten las clases, como también las personas que hacen posible que la escuela funcione y que el proceso de enseñanza y aprendizaje se efectúe de forma íntegra, al respecto la profesora Paula (T26, n. preescolar) le contó al extraterrestre lo siguiente:

la escuela es un espacio donde trabajan profesores, profesoras y personas que ayudan a cuidar a niños y niños, y además directivos que nos guían en el trabajo administrativo, es decir, en la organización de la escuela, para inyectar recursos y ver lo que no hace falta para funcionar. Aquí trabajamos todos en relación a un objetivo, esto es, que niñas y niños aprendan, se eduquen y se formen como Seres Humanos; es decir, lo que tú ves en mí, un ser humano compuesto de mente y cuerpo.

En consenso con las ideas anteriores, Maritza (T1, n. primaria), explica al extraterrestre que existe un espacio especial donde se enseña, llamado escuela, en ella hay salas con una estructura específica, "en cada una de estas salas acuden niños de edades iguales. Estando allí; se puede intercambiar ideas, experiencias, y obtener información importante del profesor que es el que guía este proceso de aprender" y añade, "Si bien estas salas son cerradas, en ellas vuelan las ideas, en un mundo mágico que le permite a nuestra especie transmitir a las nuevas generaciones todo aquello importante para la vida como ser humano". Así también lo cree el profesor Mario (T3, n. secundario)., quien indicó que en la escuela:

no solo aprenden lo que yo les enseño, sino que juegan con otros niños, comparten, comen. No solo estoy yo para enseñarle a los niños, sino que hay varios como yo, cada uno enseña sobre diferentes cosas: sobre el cuerpo, los libros, los números como también a sentarse bien, a no pelear, a escuchar, a compartir, a respetar, a ser buenas personas.

Es por este motivo que el trabajo colaborativo es importante, pues es necesario crear un clima de aula que propicie el aprendizaje de las y los estudiantes, a este respecto, la profesora Carmen (T8, n. preescolar) señaló que, en su sala de clases, los niños en general trabajan de forma autónoma, con la colaboración de la tía ayudante se ambienta la sala de acuerdo al tema de la unidad. El trabajo colaborativo también se posibilita a partir de la ayuda de los mismos estudiantes, quienes le cuentan a su profesora qué es lo que les gustaría aprender, así lo indicó la profesora Luisa (T23, n. preescolar) al decir: "me gusta que los temas de trabajo, sean propuestos por mis estudiantes, los cuales expresan en conversaciones previas al planificar mi enseñanza que es lo que les gustaría aprender, de esta manera se sienten involucrados”, y continúa diciendo:

al trabajar los niños sienten que se respeta sus ritmos, pues tratamos de que todos logren terminar y sentirse contento con lo realizado y los que presentan dificultades se les apoya, a través de algún compañero o lo hace uno de nosotros. 
Estudios Pedagógicos XLVII N 3: 27-43, 2021

HACER DE LO FAMILIAR EXTRAÑO: ANÁLISIS DE UN EJERCICIO DOCENTE RESPECTO A LAS CREENCIAS Y CONCEPCIONES QUE PROFESORAS Y PROFESORES QUE ENSEÑAN CIENCIAS TIENEN SOBRE SU LABOR...

Todo lo cual se conjuga con lo dicho por la profesora Inés (T20, n. primario), quien describió su sala de clases como un hogar, indicando que "allí sus estudiantes son importantes, pues en este espacio se les aprecia y se les motiva para ser personas con valores y hábitos". A partir de esto, es posible inferir que la percepción de hogar se amalgama con el trabajo colaborativo, pues ambos se dan de forma global dentro de la Comunidad Educativa, lo que de acuerdo a lo relatado por el profesor Mario (T3, n. secundario), se efectúa dentro de la escuela "donde hay una persona que dirige, al que llaman director, también hay personas que se ocupan de la limpieza, la comida, el orden, se llaman asistentes y estamos los profesores, quienes somos los que enseñan y también están los alumnos". De esto se puede inferir que la Comunidad Escolar en su conjunto trabaja en pos de brindar un ambiente educativo acogedor, a fin de lograr un aprendizaje significativo en las y los estudiantes.

\subsubsection{Desafío Docente: Satisfacción laboral y dificultades en su labor}

En general la labor de las y los docentes se puede ver dificultada por falta de experiencia u otras carencias que la institución educativa pueda tener. Así, lo relata la profesora Fabiola al decir, "desde un tiempo a esta parte, he aprendido bastante, pero en un principio me costó enseñar de una manera que los estudiantes se involucren en su aprendizaje" (T28, n. preescolar). A esto se suma también, carencias en la infraestructura o de tipo económicas de la institución educativa a la que pertenecen, pues tal como lo recordó la profesora Luisa (T23, n. preescolar), la falta de espacio en la sala de clases, le impiden realizar cambios significativos en el entorno,

la falta de espacio físico no me permite disponer de rincones para dar la posibilidad desarrollar la creatividad de mis estudiantes y mejorar las vivencias de su etapa de juego, por eso, al menos trato de cambiar constantemente la disposición de los elementos de la sala, cuando los niños llegan lo notan y se ven felices cuando dicen que linda esta la sala.

Sin embargo, estas dificultades se ven compensadas con el trabajo diario en el aula. Al respecto, la gran mayoría de las y los profesores participantes de esta investigación, indicaron que sentían satisfacción trabajando en la sala de clases. La principal argumentación que entregan, es que podían constatar que sus estudiantes estaban aprendiendo en un ambiente propicio y acogedor, así lo demuestra lo relatado por la profesora Inés (T20, n. primaria):

mi sala de clases es un espacio físico que está en condiciones apropiadas para que los niños y jóvenes se sientan cómodos y se ambienten a este espacio físico, es por eso, que, habitualmente se les espera con música y calefacción, con la finalidad de valorarlos como personas, para que se sientan importantes en el sistema educativo.

Se suma a ello, lo mencionado por Pamela (T6, n. primaria) al indicar que al enseñar Ciencias, ella utiliza variados materiales, con la finalidad de poder diversificar la enseñanza de las Ciencias. A este respecto, la profesora Juana (T9, n. primaria) señaló que, ella decidió incluir como herramienta didáctica a la tecnología, "he utilizado las TIC'S como una herramienta, para que mis estudiantes investiguen sobre temas científicos y las he 
sugerido a otros profesores, como al profesor de música, matemática, lenguaje y hasta el de Artes, y han subido sus rendimientos".

Estas acciones hacen posible un ambiente favorable para la enseñanza y el aprendizaje de las y los estudiantes, y, además, se propicia un ambiente grato y de cercanía entre profesor y alumno. $\mathrm{Al}$ respecto Luisa (T23, n. preescolar) argumentó:

en nuestra escuela diseñamos actividades para todos igual, desde la motivación para incitarlos a trabajar y explicar que hacemos, y también para adecuar el grado de complejidad de lo que cada uno realizará. El ambiente de comunicación lo siento muy familiar, los niños y niñas se comunican en forma muy expresiva entre ellos y siento su cercanía al dirigirse a mi cuando necesitan contarme algo, lo cual hacen con naturalidad.

Este relato se amalgama con lo declarado por el profesor Luis (T25, n. secundario), al indicar que las y los estudiantes asisten a "los colegios a aprender no solo contenidos, si no también valores y a socializar, es decir, a aceptar y compartir con otros, ellos son niños alegres, traviesos, algunos se esfuerzan más que otros en sus tareas. Otros son tímidos, ordenados" Estas descripciones nos permiten concluir que la enseñanza aborda ejes diversos, que no solo apuntan a entregar conocimientos o guiar la adquisición de aquellos, sino también a desarrollar valores y habilidades de carácter socioemocional -habilidades blandas-, que no siempre son incentivadas al entregar conocimientos, sino que se ven desarrolladas, a través, de la socialización y aceptación entre los pares.

\section{DISCUSIÓN}

Existe disparidad de visiones de los docentes con respecto a su vez los procesos de enseñanza aprendizaje, por lo que resulta difícil analizar una postura definida sobre lo que piensan de su labor profesional. Es más, se constata que sus creencias y concepciones, en ocasiones se contradicen con sus discursos y con las prácticas pedagógicas que describen al extraterrestre. Precisamente, esto ocurre por las mixturas en sus ideas, enunciados y las formas de realizar su rol profesional. Sobre este último punto, un hallazgo relevante del estudio surge al observar que las creencias y concepciones de los educadores, en ocasiones se acercan a la enseñanza tradicionalista y en otras, toman tonalidades constructivistas. Dichos elementos, ya han sido encontrados en estudios nacionales (Arancibia-Herrera et al., 2019; Orellana et al., 2018; Ravanal et al., 2018) e internacionales, sobre cogniciones docentes en diferentes disciplinas y niveles de enseñanza (Barrón, 2015; Mellado y Chaucono, 2015; Pérez et al., 2006).

Al respecto, una explicación plausible, es que el pensamiento de los educadores se sustenta en sus trayectorias biográficas-profesionales. En especial, se vincula a sus experiencias como estudiantes de enseñanza básica, secundaria y superior. Precisamente, es posible que en estas etapas de sus vidas recibieron una educación asociada a los modelos tradicionales o una amalgama entre los enfoques conductistas y constructivistas (Garritz, 2014; Inguanzo, 2010; Marcelo y Vaillant, 2013; Pajares,1992; Ramos y Casas, 2018).

En este sentido, en relación a sus creencias y cogniciones asociadas al modelo tradicional de enseñanza, se observan narrativas en las cuales se ven como dueños de los conocimientos disciplinares (Vizcaino et al., 2015), prescribiendo a los estudiantes 
a resolver determinadas tareas bajo sus explicaciones. Por lo que su labor, es convertirse en trasmisores de información, sin una mirada creativa, crítica y situada. Esto, se podría relacionar con las creencias difundidas en el profesorado de esta área, sobre la relevancia de la comprobación de los fenómenos por medio del método científico, como un camino válido, único, legítimo y necesario (García y Acevedo, 2016).

Mientras que, en otros momentos, relatan acontecimientos que se encontrarían acordes a las perspectivas teóricas del constructivismo, en las cuales el conocimiento puede ser originado desde diversas realidades al interior de cada uno de sus alumnos, con un carácter idiosincrático. Enfatizando la autonomía de estos actores, la entrega de diversas oportunidades, metodologías y recursos de enseñanza aprendizaje, las cuales consideran sus experiencias y mundo interior. Por otro lado, en los aspectos didácticos, presentan argumentos favorables sobre la enseñanza de la ciencia en pequeño grupo, las interacciones entre estudiantes a la hora de desarrollar las clases, la relevancia de las relaciones humanas (Ruiz et al., 2014) y desarrollar un buen clima y ambiente de aula. En este punto, llama la atención la relevancia que le asignan a los vínculos afectivos con sus estudiantes, quienes en muchas ocasiones los retroalimentan de una forma que los hace olvidar las dificultades propias de la profesión, como el desgaste laboral o la carencia de recursos para ejercer su labor. Esta situación ha sido estudiada por reconocidos investigadores (Day, 2006; Marcelo y Vaillant, 2013), quienes han señalado que la profesión docente tiene un componente afectivo y de relaciones humanas importantes.

En esta lógica, los aportes de los ambientes, las macro-micro políticas educativas, las historias vividas como estudiantes, las diferencias entre las concepciones proclamadas en las verbalizaciones y las acciones que se desarrollan en las escuelas, juegan un rol fundamental. Principalmente, porque si estas no se encuentran acordes a los requerimientos que los otros actores e instituciones educativas les demanda (Hidalgo \& Murillo, 2017), se corre el riesgo de provocar repercusiones en aspectos sensibles de la docencia como su construcción identitaria (Ávalos y Sotomayor, 2012; Imbernón, 2007; Marcelo y Vaillant, 2013; Vaillant, 2016).

Coincidiendo con estas ideas Vaillant (2016), señala que los docentes en su ejercicio profesional, establecen razonamientos y criterios sobre "las características esenciales de una buena enseñanza y dejan espacio a distintas maneras de ejercerla" (p. 17) dentro de los marcos referenciales que han diseñado a lo largo de su experiencia profesional. Estos, deberían ser consideradas en las políticas educativas para que las reformas en esta área logren adentrarse en el mundo interno de los profesores. Entendiendo que un enseñante no es un mero trabajador o funcionario (Cataldo, 2010) que aplica técnicas y recursos en una institución, sino que "es sujeto de su propio trabajo y actor de su pedagogía, pues es el quién modela, quien le da cuerpo y sentido en el contacto con los alumnos (negociando, improvisando, adaptando)" (Tardif, 2010, p. 109), labrando cotidianamente el currículo escolar desde sus propias elecciones y selecciones dentro de los marcos referenciales establecidos por la sociedad.

\section{CONCLUSIONES}

Las y los profesores participantes presentan visiones diversas sobre el proceso de enseñanza-aprendizaje en el ámbito de las ciencias, más allá del nivel de enseñanza en el que se desempeñan. 
En las creencias y concepciones de las y los docentes en ocasiones no se condice el discurso con la prácticas pedagógicas, ya que en ocasiones se acercan a la enseñanza tradicionalista y en otras, constructivistas.

En la práctica tradicional se constituyen como trasmisores de información, donde la comprobación de los fenómenos estudiados, está medida por el método científico.

En la arista constructivista el conocimiento puede ser originado autónomamente desde diversas realidades de cada alumno a través de la entrega de diversas oportunidades, metodologías y recursos de enseñanza-aprendizaje, considerando sus experiencias y mundo interior.

En la aproximación didáctica, se considera importante trabajar en grupos pequeños y desarrollar un buen clima de aula.

Las y los profesores aprecian como relevantes los vínculos afectivos con sus estudiantes, lo que les impulsa más allá de las dificultades propias de su quehacer docente.

\section{REFERENCIAS BIBLIOGRÁFICAS}

Arancibia-Herrera, M., Novoa-Cáceres, V. y Casanova-Seguel, R. (2019). Concepciones sobre evaluación de docentes de ciencias naturales, matemática, lenguaje e historia. Revista Educación, 43(1). https://doi.org/10.15517/revedu.v43i1.30497

Ávalos, B. y Sotomayor, C. (2012). Cómo ven su identidad los docentes chilenos. Perspectiva Educacional, 57(1), 77-95.

Barrón, C. (2015). Concepciones epistemológicas y práctica docente. Una revisión. Revista de Docencia Universitaria, (13), 35-36. https://doi.org/10.4995/redu.2015.6436

Busquets, T., Silva, M. y Larrosa, P. (2016). Reflexiones sobre el aprendizaje de las ciencias naturales: nuevas aproximaciones y desafíos. Estudios Pedagógicos, 42(especial), 117-135. https://dx.doi. org/10.4067/S0718-07052016000300010

Cataldo González, H. M. (2010). Funcionarado, política y educación. En H. Cataldo, V. Pankova, S. Ascencio y M. Sobarzo, Los confines de lo educativo (pp. 13-34). Universidad Arcis.

Day, C. (2006). Pasión por enseñar. La identidad personal y profesional del docente y sus valores. Narcea.

Denzin, N. \& Lincoln, Y. (Eds.) (2011). The Sage handbook of qualitative research. Sage.

Díaz, C., Martínez, P., Roa, I. y Sanhueza, M. (2010). Los docentes en la sociedad actual: sus creencias y cogniciones pedagógicas respecto al proceso didáctico. Polis, 9(25), 421-436. http:// dx.doi.org/10.4067/S0718-65682010000100025

Donoso, P., Rico, N. y Castro, E. (2016). Creencias y concepciones de profesores chilenos sobre las matemáticas, su enseñanza y aprendizaje. Profesorado Revista de currículum y formación del profesorado, 20(2), 76-97. https://www.redalyc.org/articulo.oa?id=56746946005

Echeverría, M., Mateos, M., Scheuer, N. y Martín, E. (2006). Enfoque del estudio de las concepciones sobre el aprendizaje y la enseñanza. En J. Pozo, N. Scheuer, M. Pérez, M. Mateos, E. Martín, y M. Cruz, Nuevas formas de pensar la enseñanza y el aprendizaje. Las concepciones de profesores y alumnos (pp. 55-89). Grao.

Fernández, M., Tuset, A., Pérez, R. y Leyva, A. (2009). Concepciones de los maestros sobre la enseñanza y el aprendizaje y sus prácticas educativas en clases de ciencias naturales. Enseñanza de las Ciencias, 27(287-298).

García, A. y Acevedo, J. (2016). Concepciones de estudiantes de profesorado de educación primaria sobre la naturaleza de la ciencia: Una evaluación diagnóstica a partir de reflexiones en equipo. Revista Mexicana de investigación educativa, 21(69), 583-610. http://www.scielo.org.mx/scielo. php?script=sci_arttext\&pid=S140566662016000200583\&lng=es\&tlng=es 
Estudios Pedagógicos XLVII N 3: 27-43, 2021

HACER DE LO FAMILIAR EXTRAÑO: ANÁLISIS DE UN EJERCICIO DOCENTE RESPECTO A LAS CREENCIAS Y CONCEPCIONES QUE PROFESORAS Y PROFESORES QUE ENSEÑAN CIENCIAS TIENEN SOBRE SU LABOR...

Garritz, A. (2014). Creencias de los profesores, su importancia y cómo obtenerlas. Educación química, 25(2), 88-92. https://doi.org/10.1016/S0187-893X(14)70529-4

Guerra, P. y Sebastián, C. (2015). Creencias epistemológicas en profesores que postulan al Programa de Acreditación de Excelencia Pedagógica: Análisis descriptivos y comparativos entre profesores que se desempeñan en los diferentes niveles de enseñanza. Estudios Pedagógicos, 41(2), 107125. http://dx.doi.org/10.4067/S0718-07052015000200007

Hidalgo, N. y Murillo, F. (2017). Las concepciones sobre el proceso de evaluación del aprendizaje de los estudiantes. Revista Iberoamericana sobre Calidad, Eficacia y Cambio en Educación, 15(1), 107-128. https://doi.org/10.15366/reice2017.15.1.007

Imbernón, F. (2007). La formación y el desarrollo profesional del profesorado. Hacia una nueva cultura profesional. Grao.

Inguanzo Arteaga, G. (2010). Creencias de los profesores de nivel de licenciatura sobre la naturaleza del conocimiento y los procesos de enseñanza aprendizaje. [Tesis doctoral, Universidas Iberoamericana de Puebla].https://rei.iteso.mx/bitstream/handle/11117/1206/6-Gonzalo-Inguanzo-Puebla. pdf? sequence $=2$

Lins Ribeiro, G. (2004). Descotidianizar. Extrañamiento y conciencia práctica, un ensayo sobre la perspectiva antropológica. En M. Boivin, A. Rosato y V. Arribas (comps.) Constructores de Otredad. Una introducción a la Antropología Social y Cultural (pp. 194-198). Editorial Antropofagia.

Lowyck, J. (1988). Pensamientos y rutinas del profesor: ¿una bifurcación? En L. M. Villar, Conocimientos, creencias y teorías de los profesores (pp. 121-133). Marfil.

Marcelo, C. y Vaillant, D. (2013). Desarrollo profesional docente ¿Cómo se aprende a enseñar? (3a edición ed.). Narcea.

Mellado, M. y Chaucono, J. (2015). Creencias pedagógicas del profesorado de una escuela rural en el contexto mapuche. Actualidades Investigativas en Educación, 15(3). http://www.scielo.sa.cr/ scielo.php?script=sci_arttext\&pid=S140947032015000300316\&lng=en\&tlng=es.

Miles, M. B. \& Huberman, A. M. (1994). Qualitative data analysis: An expanded sourcebook. Sage.

Olmos, A. (2016). Algunas reflexiones sobre la Etnografía Escolar: holismo, extrañamiento y diversidad cultural. Revista Investigación en la Escuela, 89, 1-17.

O'Reilly, M. \& Parker, N. (2014). Doing mental health research with children and adolescents: A guide to qualitative methods. Sage: London.

Orellana, C., Quintanilla, M. y Páez, R. (2018). Concepciones sobre enseñanza y aprendizaje de las Ciencias Naturales de educadoras de párvulos en formación en Chile y sus relaciones con modelos de racionalidad científica. Ciênc. Educ., Bauru, 24(4), 1029-1041. http://dx.doi. org/10.1590/1516-731320180040014

Pajares, M. (1992). Teacher's beliefs and educational research: cleaning up a messy construct. Review of Educational Research, 6(3), 307-332.

Pérez, M., Pozo, J., Pecharromán, A. y Martínez, J. C. (2006). Las concepciones de los profesores de educación secundaria sobre el aprendizaje y la enseñanza. En J. Pozo, N. Scheuer, M. Pérez, M. Mateos \& E. M. Cruz, Nuevas formas de pensar la enseñanza y el aprendizaje. Las concepciones de profesores y alumnos (pp. 231-239). Grao.

Ramos, L. y Casas, L. (2018). Concepciones y creencias de los profesores de Honduras sobre enseñanza, aprendizaje y evaluación de las matemáticas. Revista Latinoamericana de Investigación en Matemática Educativa, 21(3), 275-299. https://doi.org/10.12802/relime.18.2132

Ravanal, E., López, F. y Rodríguez, L. (2018). Creencias de profesores chilenos de biología sobre la preparación de la enseñanza. Revista Eureka sobre Enseñanza y Divulgación de las Ciencias, 15(3), 3601. https://doi.org/10.25267/Rev_Eureka_ensen_divulg_cienc.2018.v15.i3.3601

Reimers, F. y Chung, C. (2016). Introducción. Estudio comparativo de los propósitos de la educación en el siglo XXI. En F. Reimers y C. Chung (Edits.), Enseñanza y aprendizaje en el siglo XXI. Metas, políticas educativas y currículo en seis países (pp. 13-35). Fondo de la Cultura Económica. 

CONCEPCIONES QUE PROFESORAS Y PROFESORES QUE ENSEÑAN CIENCIAS TIENEN SOBRE SU LABOR...

Ruffinelli, A. (2016). Ley de desarrollo profesional docente en Chile: de la precarización sistemática a los logros, avances y desafíos pendientes para la profesionalización. Estudios Pedagógicos, 42(4), 261-279. http://dx.doi.org/10.4067/S0718-07052016000500015

Ruiz, F., Marquéz, C. y Tamayo, Ó. (2014). Cambios en las concepciones de los docentes sobre la argumentación y su desarrollo en clase de ciencias. Enseñanza de las Ciencias, 32(3), 5370. http://dx.doi.org/10.5565/rev/ensciencias.985

Shön, D. (1992). La formación de profesionales reflexivos. Paidos.

Schunk, D. (2012). Conductismo. En D. Schunk, Teorías de aprendizaje. Una perspectiva Educativa (6 $6^{\mathrm{a}}$ ed.) (pp. 71-116). Pearson educación.

Shulman, L. S. (1989). Paradigmas y programas de investigación en el estudio de la enseñanza: una perspectiva contemporánea. En M. Wittrock, La investigación de la enseñananza I. Enfoques, teorías y métodos (pp. 9-84). Paidós.

Tardif, M. (2010). Los saberes del docente y su desarrollo profesional. Narcea.

Vaillant, D. (2016). El fortalecimiento del desarrollo profesional docente: una mirada desde Latinoamérica. Journal of supranational policies of education, (5), 5-21. http://dx.doi. org/10.15366/jospoe2016.5

Vizcaíno, A., Cadalso, A. y Manzano, M. (2015). Adaptación de un cuestionario para evaluar las creencias epistemológicas sobre la matemática de profesores de secundaria básica. Revista Complutense de Educación, 26(2), 255-273. https://doi.org/10.5209/rev_RCED.2015.v26. n2.42981 
